\title{
Elicitation of Isatis tinctoria L. hairy root cultures by salicylic acid and methyl jasmonate for the enhanced production of pharmacologically active alkaloids and flavonoids
}

\author{
Qing-Yan Gai ${ }^{1}$ Jiao Jiao ${ }^{1} \cdot$ Xin Wang ${ }^{1} \cdot$ Yu-Ping Zang ${ }^{1} \cdot \mathrm{Li}-\mathrm{Li} \mathrm{Niu}{ }^{1} \cdot$ Yu-Jie Fu ${ }^{2}$
}

Received: 19 April 2018 / Accepted: 29 December 2018 / Published online: 3 January 2019

(c) Springer Nature B.V. 2019

\begin{abstract}
Plant cell and organ cultures via the implementation of effective elicitation strategies can offer attractive biotechnological platforms for the enhanced production of phytochemicals of pharmaceutical interest. For the first time, the elicitation of exogenous signal molecules was conducted to enhance the production of pharmacologically active alkaloids and flavonoids in Isatis tinctoria L. hairy root cultures (ITHRCs). ITHRCs III and V correspondingly possessing high alkaloid and flavonoid productivity were adopted for elicitation treatments. The maximum accumulation of alkaloids in ITHRCs III elicited by $142.61 \mu \mathrm{M}$ salicylic acid for $28.18 \mathrm{~h}$ and flavonoids in ITHRCs V elicited by $179.54 \mu \mathrm{M}$ methyl jasmonate for $41.87 \mathrm{~h}$ increased 5.89- and 11.21-folds as compared with controls, respectively. Moreover, expressions of 11 genes involved in alkaloid and flavonoid biosynthetic pathways were significantly up-regulated following elicitation, among which $Y U C C A, C H I$ and $F 3^{\prime} H$ genes might play a crucial role in the target phytochemical augmentation. Overall, two effective elicitation protocols were provided here to improve the yields of bioactive alkaloids and flavonoids in ITHRCs, which was useful for the scale-up production of these valuable compounds to meet the demands for natural bioactive ingredients by pharmaceutical industries.
\end{abstract}

Keywords Elicitation · Isatis tinctoria L. · Hairy root cultures $\cdot$ Bioactive phytochemicals · Yield enhancement

\section{Introduction}

Communicated by Wagner Campos Otoni.

Qing-Yan Gai and Jiao Jiao have contributed equally to this work.

Key Message The elicitation of exogenous signal molecules was conducted to enhance the production of pharmacologically active alkaloids and flavonoids in Isatis tinctoria L. hairy root cultures for the first time.

Electronic supplementary material The online version of this article (https://doi.org/10.1007/s11240-018-01553-8) contains supplementary material, which is available to authorized users.

Jiao Jiao

jj_nefu@163.com

$\triangle$ Yu-Jie Fu

yujie_fu@163.com

1 Key Laboratory of Forest Plant Ecology, Ministry of Education, Northeast Forestry University, Harbin 150040, People's Republic of China

2 Beijing Advanced Innovation Center for Tree Breeding by Molecular Design, Beijing Forestry University, Beijing 100083, People's Republic of China
The dried roots of Isatis tinctoria L. (Radix isatidis), one of the top-selling herbal medicines in East Asian and United States (Hamburger 2002), are highly appreciated for their notable anti-influenza efficacy, such as severe acute respiratory syndrome (SARS) and novel swine-origin influenza A (H1N1) (Lin et al. 2005; Wang et al. 2011). Alkaloids and flavonoids present in Radix isatidis are thought to be the primary pharmacological active constituents, which probably contribute to the outstanding antiviral activity of this herbal medicine (Hamburger 2002; Lin et al. 2005; Nguyen et al. 2017). However, the field cultivation of I. tinctoria is associated with an important challenge that the phytochemical profile is highly affected by geographical regions, climatic fluctuations, and soil conditions, which can significantly affect the therapeutic efficacy of Radix isatidis (Chen et al. 2015).

Currently, plant cell and organ culture technology has been well regarded as an attractive alternative for the sustainable production of phytochemicals of pharmaceutical interest with uniform quality (Murthy et al. 2014). In this 
context, we have developed an in vitro culture system, i.e. I. tinctoria hairy root cultures (ITHRCs), as an alternative to the field cultivation for the sustainable and standard production of bioactive alkaloids and flavonoids (Gai et al. 2015a, b). However, search of cost-effective strategies that can further enhance alkaloid and flavonoid accumulation in ITHRCs is an essential task, given the possibility of exploiting this biotechnological system to produce pharmaceutical phytochemicals in industrially viable processes.

Strategies including genetic modification, elicitation, permeabilization, immobilization, two phase system, etc., have been successfully employed to enhance the biotechnological production of high-added value compounds in various plant in vitro cultures, among which elicitation via application of biotic/abiotic elicitors for activating plant defense reactions is implemented extensively owing to its low cost and simplicity of usage (Isah et al. 2018). Notably, alkaloids and flavonoids are always regarded as phytoalexins that can be inducibly synthesized by environmental stresses, aiming to protect plants against unfavorable conditions (Ahuja et al. 2012). Based on this principle, application of external elicitors is likely to promote alkaloid and flavonoid accumulation in ITHRCs via inducing plant defense responses.

Generally, natural phytohormones such as salicylic acid (SA), acetylsalicylic acid (ASA), and methyl jasmonate (MJ), are involved in the signal transduction cascades of plant defense responses (Giri and Zaheer 2016; RamirezEstrada et al. 2016). This character makes them act as potent elicitors when added exogenously in culture media for the enhanced production of defensive phytochemicals in various plant in vitro culture systems (Giri and Zaheer 2016; Narayani and Srivastava 2017; Ramirez-Estrada et al. 2016). Moreover, it is worth mentioning that SA, ASA, and MJ can be produced by plants during their normal metabolism processes (Narayani and Srivastava 2017). In view of the bio-safety of products, the utilization of these atoxic molecules as elicitors for improving phytochemical yield in plant in vitro cultures is strongly recommended.

In the present study, the elicitation effects of SA, ASA, and $\mathrm{MJ}$ on alkaloid and flavonoid accumulation in two high-productive ITHRCs (ITHRCs III and V) were initially compared. To achieve the maximum alkaloid and flavonoid yield, the elicitation conditions of the selected elicitors were carefully optimized by central composite designs. Also, the profiles of six alkaloids and eight flavonoids before and after the optimal elicitation were evaluated. Moreover, transcriptional profiles of associated genes involved in alkaloid and flavonoid biosynthetic pathways were investigated to shed light on the molecular mechanism underlying elicitation. To the best of our knowledge, there are no reports regarding the application of the exogenous signal molecules (SA, ASA, and $\mathrm{MJ}$ ) to elevate the production of pharmacologically active alkaloids and flavonoids in ITHRCs.

\section{Experimental}

\section{Hairy root cultures}

Isatis tinctoria L. hairy roots were previously induced from petiole explants by Agrobacterium rhizogenes LBA9402 mediated transformation in our laboratory, and two lead root lines, i.e. ITHRL III (high alkaloid productivity) and ITHRL V (high flavonoid productivity), were adopted in this study for elicitation experiments (Gai et al. 2015a, b). The two stock lines (ITHRL III and V) were maintained on phytohormone-free MS/2-based solid medium supplemented with $30 \mathrm{~g} / \mathrm{L}$ sucrose at $25 \pm 1{ }^{\circ} \mathrm{C}$ in the dark. Moreover, ITHRCs III and V were obtained by correspondingly inoculating ITHRL III and V (inoculum size 0.75\%) into $250 \mathrm{~mL}$ Erlenmeyer flask containing $150 \mathrm{~mL}$ of MS/2 liquid medium under the optimal culture conditions described in our previous studies (Gai et al. 2015a, b).

\section{ITHRCs treated by different elicitors}

In this work, ITHRCs III and V undergone the optimal culture conditions were subjected to elicitation treatments, which aimed to enhancing the phytochemical accumulation without affecting their biomass productivity. The elicitors used in this work (SA, ASA and MJ) were dissolved in absolute ethanol and filter-sterilized before use. Prior to elicitation, the spent media of ITHRCs III (23 day-old) and ITHRCs V (24 day-old) were renewed by fresh ones, in which different elicitors with various concentrations $(50-400 \mu \mathrm{M})$ were added. During elicitation, all elicitortreated ITHRCs were incubated with a series of durations $(12-96 \mathrm{~h})$ on a rotary shaker $(120 \mathrm{rpm})$ at $25 \pm 1{ }^{\circ} \mathrm{C}$ in the dark. For control, non-treated and ethanol-treated ITHRCs (addition of ethanol without any elicitor) underwent the same conditions as the experimental groups. After elicitation, hairy roots were collected by filtration, and rinsed by distilled water. For phytochemical extraction, part of roots were dried in a vacuum oven and ground into fine powders. For RNA extraction, the rest of roots were frozen immediately with liquid nitrogen and stored at $-80{ }^{\circ} \mathrm{C}$. Also, the culture media were collected for the liquid-liquid extraction of phytochemicals.

\section{Liquid chromatographic tandem mass spectrometry (LC-MS/MS) analysis}

The complete extraction of phytochemicals from hairy roots was performed as described previously (Gai et al. 2015a, b). Phytochemicals in culture media were extracted twice by phase partitioning with ethyl acetate, and the organic phase 
was condensed to dryness using a rotary evaporator under vacuum. Prior to LC-MS/MS analysis, extracts obtained from hairy roots and culture media were re-dissolved in acetonitrile $(20 \mathrm{~mL})$ and filtered through a nylon filter $(0.45 \mu \mathrm{m})$. Six alkaloids (epigoitrin, isatin, indole-3-carboxaldehyde, tryptanthrin, indigo, and indirubin) and eight flavonoids (rutin, neohesperidin, buddleoside, liquiritigenin, quercetin, isorhamnetin, kaempferol, and isoliquiritigenin) in the extracts were determined using an Agilent 1100 series HPLC (Agilent Technologies, USA) coupled to an API 3000 triple tandem quadrupole MS (Applied Biosystems, Canada). The detailed operational procedures were carried out as reported previously (Gai et al. 2015a, b). MS selected reaction monitoring (SRM) mode with precursor ion/product ion combinations of $\mathrm{m} / \mathrm{z} 127.8 \rightarrow 58.0, \mathrm{~m} / \mathrm{z} 145.6 \rightarrow 118.0, \mathrm{~m} / \mathrm{z}$ $143.9 \rightarrow 115.1, \mathrm{~m} / \mathrm{z} 247.2 \rightarrow 218.9, \mathrm{~m} / \mathrm{z} 261.0 \rightarrow 217.0, \mathrm{~m} / \mathrm{z}$ $261.2 \rightarrow 157.0, \mathrm{~m} / \mathrm{z} 609.1 \rightarrow 300.0, \mathrm{~m} / \mathrm{z} 609.5 \rightarrow 301.4, \mathrm{~m} / \mathrm{z}$ $591.4 \rightarrow 283.1, \mathrm{~m} / \mathrm{z} 255.9 \rightarrow 119.0, \mathrm{~m} / \mathrm{z} 301.0 \rightarrow 151.0, \mathrm{~m} / \mathrm{z}$ $315.0 \rightarrow 300.1, \mathrm{~m} / \mathrm{z}, 285.3 \rightarrow 183.1$, and $\mathrm{m} / \mathrm{z}, 255.4 \rightarrow 118.9$ was conducted for the quali-quantitative analysis of epigoitrin (EPI), isatin (ISA), indole-3-carboxaldehyde (INC), tryptanthrin (TRY), indigo (ING), indirubin (INR), rutin (RUT), neohesperidin (NEO), buddleoside (BUD), liquiritigenin (LIQ), quercetin (QUE), isorhamnetin (ISR), kaempferol (KAE), and isoliquiritigenin (ISL), respectively. The analyte content was reported as the microgram per gram of hairy roots (DW).

\section{Quantitative real-time PCR (qRT-PCR) analysis}

A MiniBEST Plant RNA Extraction Kit (TaKaRa, China) was used to extract total RNA from the frozen hairy roots ( $250 \mathrm{mg}$, fresh weight) following the manufacturer's guidelines. After the quality and quantity assessment, a certain amount of RNA (500 ng) was reverse-transcribed to cDNA using a PrimeScript ${ }^{\mathrm{TM}} \mathrm{RT}$ reagent Kit (TaKaRa, China) in accordance with the manufacturer's protocols. The synthesized cDNA $(20 \mu \mathrm{L})$ was diluted with sterile water to $200 \mu \mathrm{L}$, and stored at $-20{ }^{\circ} \mathrm{C}$ till qRT-PCR assay. Specific primers of associated genes involved in alkaloid and flavonoid biosynthetic pathways (Table 1) were designed based on the reported transcriptome sequences (Chen et al. 2013; Tang et al. 2014). The qRT-PCR analysis of all target genes was performed on a Stratagene Mx3000P Real-Time PCR system (Agilent Technologies, USA) using SYBR Green detection chemistry. The cycling procedure was determined as follows: initial denaturation step of $95{ }^{\circ} \mathrm{C}$ for $3 \mathrm{~min}$, followed by 40 amplification cycles with denaturation at $95^{\circ} \mathrm{C}$ for $15 \mathrm{~s}$, annealing at $55^{\circ} \mathrm{C}$ for $30 \mathrm{~s}$, and extension at $72^{\circ} \mathrm{C}$ for $20 \mathrm{~s}$. The relative expression levels of all target genes were quantified based on Ubiquitin (the internal reference gene) according to the ${ }^{\Delta \Delta} \mathrm{C}_{\mathrm{T}}$ method (Livak and Schmittgen 2001).
Table 1 Specific primers for alkaloid and flavonoid biosynthetic genes

\begin{tabular}{ll}
\hline Gene names & Primer sequences $\left(5^{\prime}-3^{\prime}\right)$ \\
\hline AADC & Forward: GCGAGCAGCAATCATAACAA \\
& Reverse: CCTGAAACGGTGGAACAAGT \\
IPDC & Forward: TCCAAAAGCTCCTTGCTTGT \\
& Reverse: CAAACAAGCGTGTCATTGCT \\
YUCCA & Forward: AGAATGGTTTCGGTCACGTC \\
& Reverse: CGTGTCTCTCCCTTCTTCG \\
IAMT & Forward: CGTCCGCTTCAACTACTTCC \\
& Reverse: AGAGCGTGACGGATAGGAGA \\
PAL & Forward: TCACTCCTTCACTCCCTCTC \\
& Reverse: TGATTCCCGCCATCTTGAAG \\
F4H & Forward: AATCGACACGGTTCTTGGAC \\
& Reverse: GCTTAGCGTCGTTGAGGTTC \\
$4 C L$ & Forward: TTGCCGATGTTCCACATCTA \\
& Reverse: GAGCAGCACCAGATTCACA \\
CHS & Forward: GGGATAAGCGACTGGAACTC \\
& Reverse: GAAACCAAACAAGACACCCC \\
CHI & Forward: CTTCCATCCTCTTCGCTCTC \\
FS & Reverse: CAAGACTCAACCTAGCTCCAG \\
Fbiquitin & Forward: CCACCTCATATCAGCGATCAAG \\
& Reverse: GATTACAGGGAGGTGAACGAG \\
& Forward: TCAAACACGTTGCACCAAAT \\
& Reverse: GACTGCTTCTCCATCCAAGC \\
& Forward: ACCCTCACGGGGAAGACCATC \\
& Reverse: ACCACGGAGACGGAGGACAAG \\
\hline &
\end{tabular}

\section{Statistical analysis}

All experiments were conducted in triplicate, and results were given as averages \pm standard deviations. All statistical analyses were carried out using the SPSS statistical software 17.0 (SPSS Inc, Chicago, USA) and the Design-Expert Software Version 11 (State-Ease, Inc., Minneapolis, MN). One-way analysis of variance with Tukey's test was used to determine significant differences between multiple groups of data at $P$ values $<0.05$.

\section{Results and discussion}

\section{Effect of different elicitors on alkaloid and flavonoid accumulation}

As reported previously, ITHRCs III (high alkaloid productivity) and ITHRCs III (high flavonoid productivity) cultured at days 24 and 25 were capable of producing the maximum phytochemicals and biomasses (Gai et al. 2015a, b). In an attempt to further enhance the target phytochemical yield without affecting the biomass productivity, the exogenous 
addition of MJ, SA, and ASA was conducted to treat 24 dayold ITHRCs III and 25 day-old ITHRCs V for investigating their elicitation effects on the accumulation of alkaloids (total yields of EPI, ISA, INC, TRY, ING, and INR) and flavonoids (total yields of RUT, NEO, BUD, LIQ, QUE, ISR, KAE, and ISL), respectively.

It was clearly observed from Fig. 1 that different types of elicitors exhibited distinct influences on the phytochemical accumulation in ITHRCs. This indicated that the sensitivity of alkaloid or flavonoid biosynthesis toward elicitation varied with the elicitors used in ITHRCs. Generally, plant in vitro cells/organs can specifically perceive different types of elicitors and trigger diverse signal transduction pathways, thus eventually resulting in accumulating distinct levels of defensive secondary metabolites (Giri and Zaheer 2016; Narayani and Srivastava 2017). In comparison with nontreated control, the significant improvements of alkaloid yield (5.42-, 4.38-, and 2.11-fold increases) were obtained when SA, ASA, and MJ $(100 \mu \mathrm{M})$ were fed in ITHRCs III for $24 \mathrm{~h}$, respectively (Fig. 1a). And, the significant enhancements of flavonoid production (4.36-, 3.77-, and 8.25-fold increases) were found when ITHRCs $\mathrm{V}$ was subjected to
SA, ASA, and MJ (100 $\mu \mathrm{M})$ for $24 \mathrm{~h}$, respectively (Fig. 1c). Accordingly, SA was more effective for promoting alkaloid accumulation in ITHRCs III than MJ and ASA, and MJ was found to be superior for improving flavonoid yield in ITHRCs V as against SA and ASA. Moreover, ethanol (the solvent used to dissolve elicitors) could slightly improve the target phytochemical yields as against non-treated control. This phenomenon was consistent with the previous reports in which ethanol was also described as an elicitor (el JaberVazdekis et al. 2008; Venugopalan and Srivastava 2015).

As shown in Fig. 1a-d, SA or MJ within the concentration range of 50-200 $\mu \mathrm{M}$ together with the elicitation duration of $12-48 \mathrm{~h}$ was found to favor the accumulation of alkaloids or flavonoids in ITHRCs. Similarly, when exogenously applied to various plant in vitro cultures, the concentration of MJ or SA within the range of 100-200 $\mu \mathrm{M}$ positively stimulates the workflow of secondary biosynthetic pathways during the period of $24-96 \mathrm{~h}$, leading to the elevated production of secondary metabolites as diverse as flavonoids, alkaloids, terpenoids, glucosinolates and lignans (Giri and Zaheer 2016; Ramirez-Estrada et al. 2016). To achieve the maximum alkaloid and flavonoid accumulation in ITHRCs,
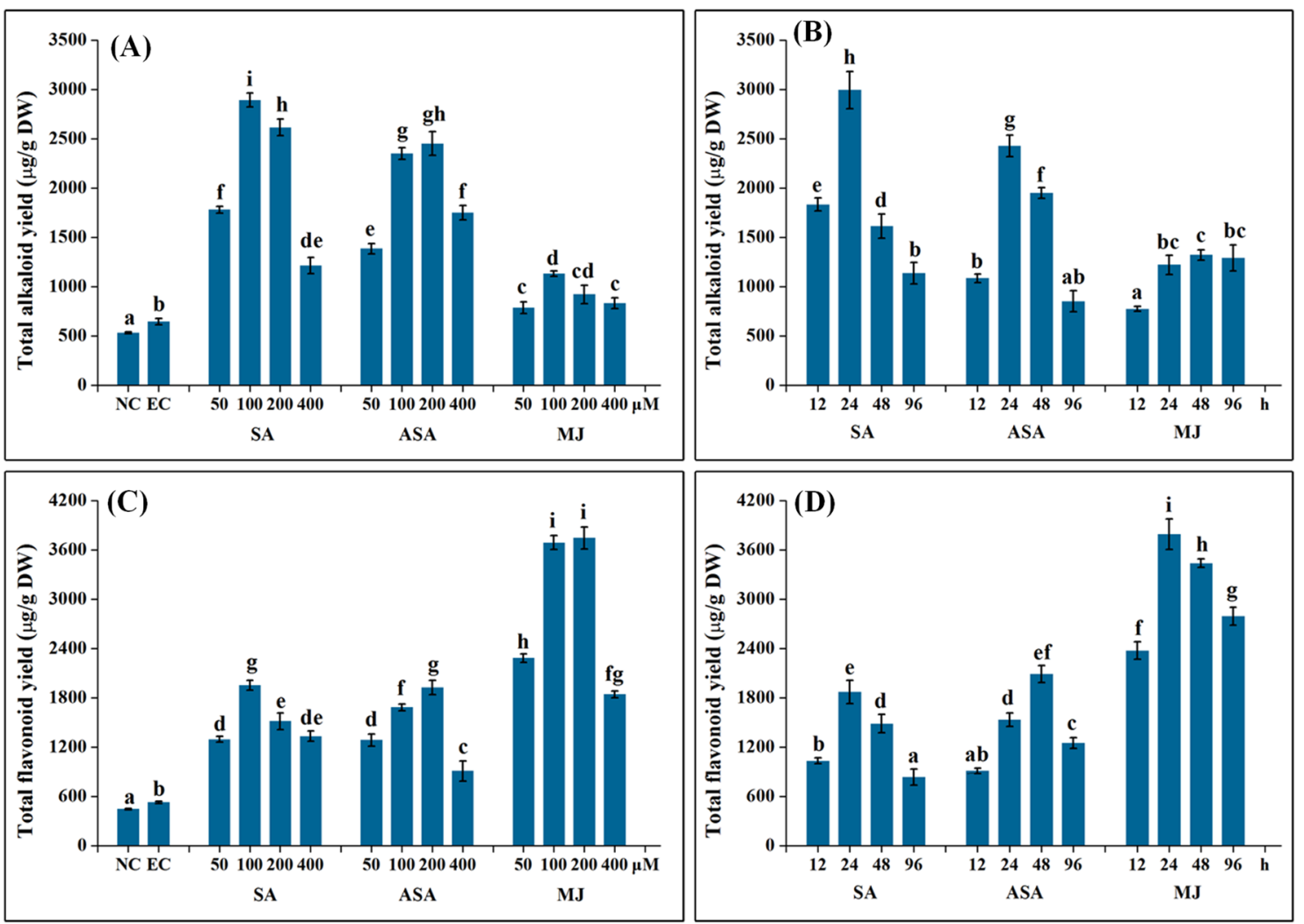

Fig. 1 Elicitation effects of SA, ASA and MJ with different concentrations $(50,100,200$, and $400 \mu \mathrm{M})$ on total alkaloid yield in 23 dayold ITHRCs III (a) and total flavonoid yield in 24 day-old ITHRCs $\mathrm{V}$ (c) under exposure duration $24 \mathrm{~h}$. Elicitation effects of SA, ASA and $\mathrm{MJ}$ with the same elicitor concentration $(100 \mu \mathrm{M})$ on total alka- loid yield in 23 day-old ITHRCs III (b) and total flavonoid yield in 24 day-old ITHRCs V (d) under different exposure durations (12, 24, 48, and 96 h). $N C$ non-treated control, EC ethanol-treated control. Mean \pm SD values not sharing the same lowercase letters are significantly different $(P<0.05)$ 
MJ and SA elicitation requires further studies to find their optimal concentration and exposure time.

\section{Optimization of elicitation conditions for alkaloid and flavonoid production}

Recently, statistical experimental design is widely adopted as an effective tool for the process optimization to improve the productivity of phytochemicals and biomasses in plant in vitro cultures, which can accurately predict the values of input parameters on a given output in a cost efficient manner (Schmitz et al. 2016). In this regard, central composite designs based mathematical modeling were adopted to optimize variables (SA or MJ concentration of $50-200 \mu \mathrm{M}$ and exposure time of 12-48 h) on dependent variables (total yields of alkaloids or flavonoids) in this work. Table $\mathrm{S} 1$ and Table S2 illustrate the experimental design matrixes for alkaloid and flavonoid accumulation in the elicited ITHRCs III and $\mathrm{V}$, respectively.

As ANOVA results shown in Table $\mathrm{S} 3$ and Table $\mathrm{S} 4$, the significances of the built mathematical models $(P<0.0005)$, non-significances of "lack of fit" $(P>0.05)$ and acceptable determination coefficients $\left(R^{2} \geq 0.9334\right)$ indicated that the established quadratic models were applicable. The following equations were used to express the yields of alkaloids and flavonoids in the elicited ITHRCs, respectively.

$$
\begin{aligned}
Y_{\mathrm{TAK}}= & 3144.83+377.21 X_{1}-132.08 X_{3}-183.61 X_{1} X_{3} \\
& -844.49 X_{1}^{2}-864.14 X_{3}^{2}
\end{aligned}
$$

$$
\begin{aligned}
Y_{\mathrm{TFL}}= & 4333.27+913.36 X_{2}+832.97 X_{3}+343.03 X_{2} X_{3} \\
& -784.33 X_{2}^{2}-819.59 X_{3}^{2}
\end{aligned}
$$

where $Y_{\text {TAK }}$ was the total yields of EPI, ISA, INC, TRY, ING, and INR ( $\mu \mathrm{g} / \mathrm{g} \mathrm{DW}) ; Y_{\mathrm{TFL}}$ was the total yields of RUT, NEO, BUD, LIQ, QUE, ISR, KAE, and ISL ( $\mu \mathrm{g} / \mathrm{g}$ DW); $X_{1}$ was the concentration of $\mathrm{SA}(\mu \mathrm{M}) ; X_{2}$ was the concentration of $\mathrm{MJ}(\mu \mathrm{M}) ; X_{3}$ was the exposure time (h).

As the response contour shown in Fig. 2A, the alkaloid yield increased significantly with the synergistic increase of SA concentration and exposure time. However, the alkaloid yield was observed to decrease when SA concentration exceeded $150 \mu \mathrm{M}$ or elicitation time went beyond $30 \mathrm{~h}$. Similarly, the flavonoid yield exhibited a decline trend under high MJ concentration $(175-200 \mu \mathrm{M})$ or long duration (42-48 h) (Fig. 2b). Generally, plants exposed to appropriate elicitation conditions can induce the eustress that contributes to the activation of plant defense secondary metabolisms for self-protection, and when the exposure exceeds the tolerance-limit level, plants will suffer from the distress that is characterized by metabolic
(A)

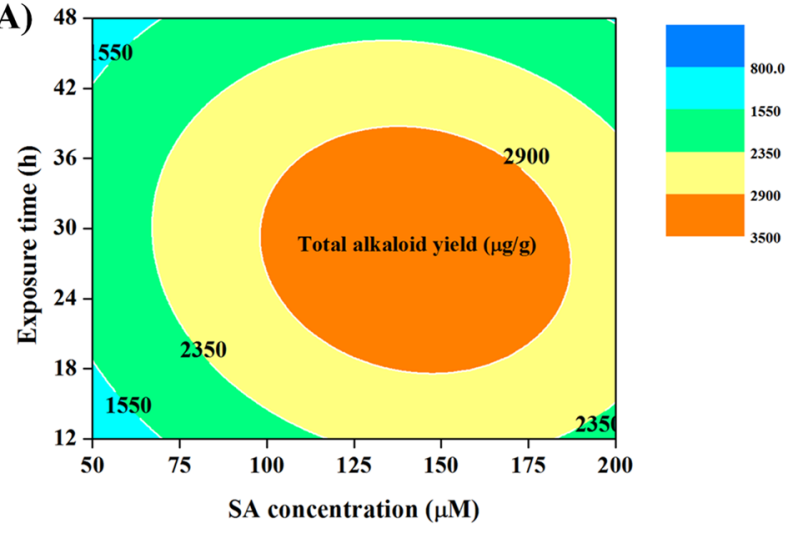

(B)

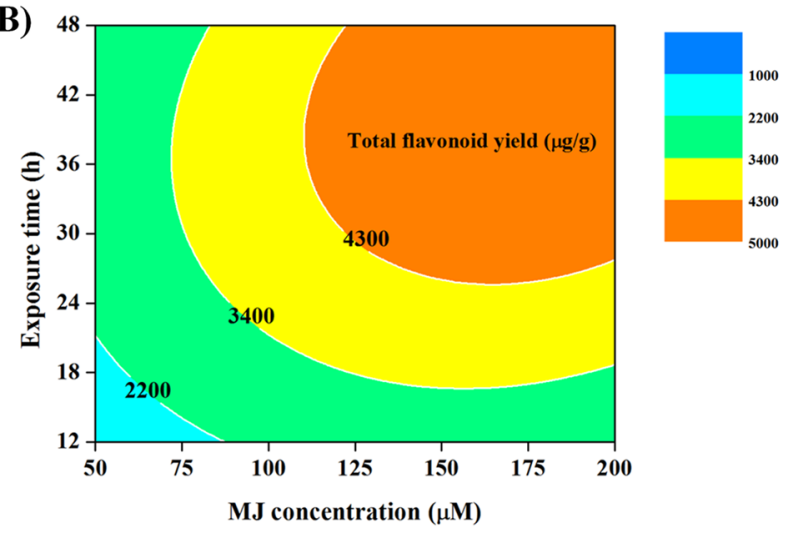

Fig. 2 a Response contour of varying SA concentration (50-200 $\mu$ M) and exposure duration (12-48 h) on total alkaloid yield in 23 day-old ITHRCs III. b Response contour of varying MJ concentration (50$200 \mu \mathrm{M})$ and exposure duration (12-48 h) on total flavonoid yield in 24 day-old ITHRCs V

damage or cell death, thus leading to the reduced productivity of secondary metabolites (Kranner et al. 2010). Additionally, plants have thresholds for specific secondary metabolites that can be synthesized by themselves. Once the secondary metabolites are accumulated at high levels, plants will be stimulated to generate enzymes for product degradation through the feedback regulation (Malik et al. 2013).

Based on the aforementioned models, the optimal elicitation conditions for alkaloid accumulation in ITHRCs III were SA concentration $142.61 \mu \mathrm{M}$ and elicitation time $28.18 \mathrm{~h}$, and the optimal elicitation conditions for flavonoid accumulation in ITHRCs $\mathrm{V}$ were $\mathrm{MJ}$ concentration $179.54 \mu \mathrm{M}$ and elicitation time $41.87 \mathrm{~h}$. The actual yields of total alkaloids and total flavonoids under the forecasted parameters were $3146.55 \pm 97.63 \mu \mathrm{g} / \mathrm{g}$ DW in ITHRCs III and $4963.15 \pm 110.18 \mu \mathrm{g} / \mathrm{g}$ DW in ITHRCs III, which fit well with the corresponding values ( $3195.72 \mu \mathrm{g} / \mathrm{g}$ DW and $4940.08 \mu \mathrm{g} / \mathrm{g}$ DW) predicted by the above theoretical models, respectively. Consequently, the elicitation conditions optimized by central composite designs were reliable. 


\section{Profiles of alkaloid and flavonoid derivatives following elicitation}

To investigate the changes in profiles of six alkaloids and eight flavonoids following the optimal elicitation, extracts from SA-elicited ITHRCs III and MJ-elicited ITHRCs V were determined by LC-MS/MS with SRM mode (Fig. 3a, b), and the yields of all target compounds calculated are presented in Fig. 3c, d. In detail, the contents of six alkaloid derivatives i.e. EPI, ISA, INC, TRY, ING, and INR in SA-elicited ITHRCs III were 5.93-, 7.46-, 3.18-, 10.84-, 7.34-, and 5.97-fold higher than those in control, respectively. And the levels of eight flavonoid derivatives i.e. RUT, NEO, BUD, LIQ, QUE, ISR, KAE, and ISL in MJ-elicited ITHRCs V were 5.38-, 13.29-, 4.71-, 6.13-, 15.97-, 9.58-, 14.63-, and 5.81-fold higher relative to those in control, respectively. Overall, the total alkaloid yield $(3146.55 \pm 97.63 \mu \mathrm{g} / \mathrm{g} \mathrm{DW})$ in SA-elicited ITHRCs III and the total flavonoid yield $(4963.15 \pm 110.18 \mu \mathrm{g} / \mathrm{g}$ DW) in MJ-elicited ITHRCs $\mathrm{V}$ increased 5.89- and 11.21-folds as against those in the corresponding controls $(533.84 \pm 13.02 \mu \mathrm{g} / \mathrm{g}$ DW and $442.63 \pm 9.46 \mu \mathrm{g} / \mathrm{g} \mathrm{DW})$, respectively.
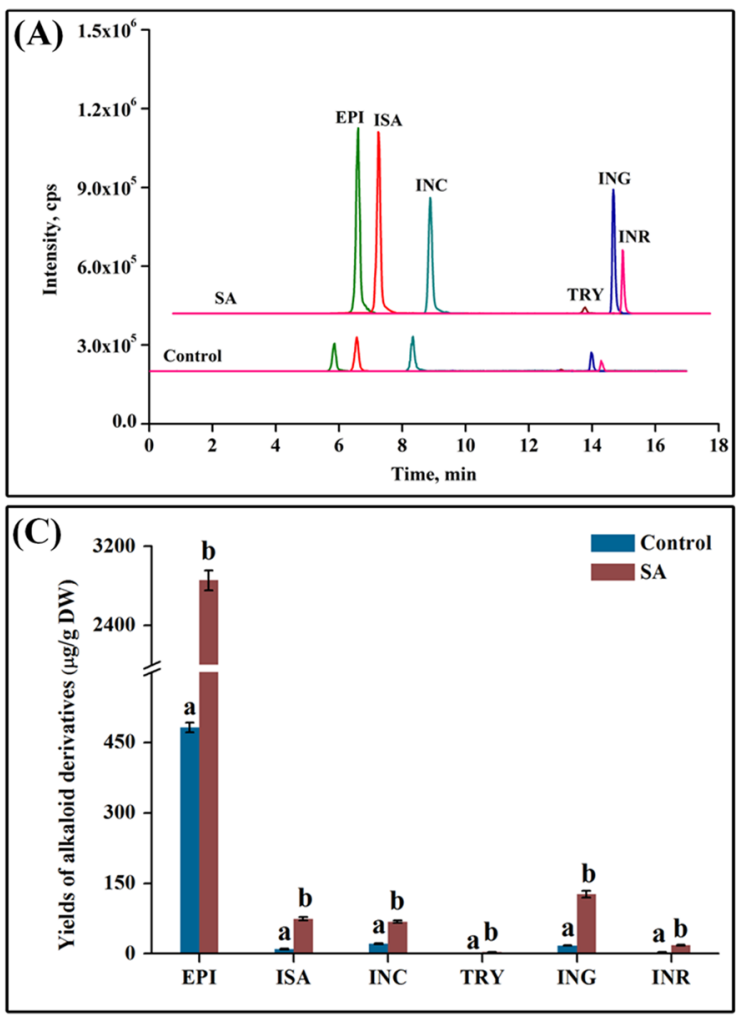

Fig. 3 Representative LC-MS/MS chromatograms of alkaloids in extracts form SA-elicited ITHRCs III (a) and flavonoids in extracts form MJ-elicited ITHRCs V (b). Yields of six alkaloid derivatives in extracts form SA-elicited ITHRCs III (c) and eight flavonoid
The treatment of plant in vitro cultures with exogenous $\mathrm{SA}$ or MJ is able to active diverse effectors followed by amplification of the generated secondary signals through a cascade of transducers. And this can lead to a variety of defense responses in which the activation of genes associated with phytoalexin biosynthesis is flagged as a characteristic even (Giri and Zaheer 2016; Narayani and Srivastava 2017; Ramirez-Estrada et al. 2016). As inferred, the enhanced accumulation of alkaloids and flavonoids in the elicited ITHRCs might be ascribed to the activation of genes related to alkaloid and flavonoid biosynthesis. Thus, transcriptional analysis of genes involved in alkaloid and flavonoid biosynthetic pathways is necessary in the following studies.

\section{Biosynthetic gene expression underlying elicitation}

To further shed light on the molecular response mechanism in ITHRCs underlying SA and MJ elicitation, expressions of 11 genes encoding enzymes involved in alkaloid and flavonoid biosynthetic pathways were determined by qRT-PCR, i.e. aromatic amino acid decarboxylase (AADC), indole pyruvate decarboxylase (IPDC), YUCCA monooxygenase
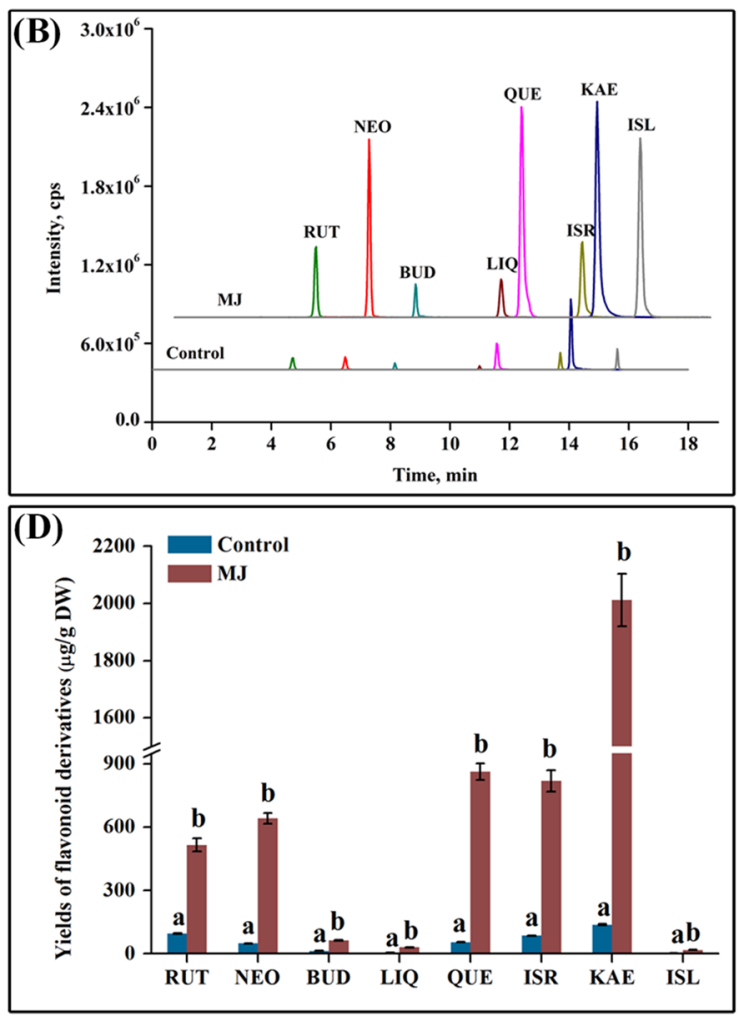

derivatives in extracts form MJ-elicited ITHRCs V. Mean \pm SD values not sharing the same lowercase letters are significantly different $(P<0.05)$ 
(YUCCA), indole-3-acetate methyl transferase (IAMT), phenylalanine ammonia lyase (PAL), cinnamate-4-hydroxylase (C4H), 4-coumarate coenzyme A ligase (4CL), chalcone synthase (CHS), chalcone isomerase (CHI), flavonol synthase (FS) and flavonoid 3'-hydroxylase (F3'H). In this work, samples collected from SA-elicited ITHRCs III at 12, 28.18 and $48 \mathrm{~h}$ were taken for qRT-PCR analysis of alkaloid biosynthetic genes (AADC, IAMT, IPDC and YUCCA). And samples obtained from MJ-elicited ITHRCs V at 12, 41.87 and $48 \mathrm{~h}$ were applied for qRT-PCR analysis of flavonoid biosynthetic genes (PAL, C4H, 4CL, CHS, CHI, F3' $\mathrm{H}$ and $F S$ ).

As shown in Figs. 4 and 5, transcription levels of all investigated genes were significantly up-regulated in ITHRCs undergoing SA and MJ elicitation. Moreover, the expression profiles of $A A D C, Y U C C A, 4 C L, C H S, C H I$ and $F 3^{\prime} H$ were found to be positively correlated with the accumulation patterns of alkaloids or flavonoids. Additionally, it is clearly observed from Fig. 4 that YUCCA gene exhibited the greatest transcriptional abundance (30.98-fold) at the optimal elicitation time point $(28.18 \mathrm{~h})$ necessary for the maximal alkaloid production, which suggested that this gene might be more sensitive and crucial for inducing alkaloid biosynthesis in ITHRCs III following SA elicitation. Moreover, the transcriptional levels of $\mathrm{CHI}$ (34.07-fold) and $F 3^{\prime} \mathrm{H}$ (20.91-fold) at $41.87 \mathrm{~h}$ (the time point where flavonoid yield was the highest) were found to be much higher than other flavonoid biosynthetic genes (Fig. 5), indicating that the both genes might be more responsible for flavonoid biosynthesis in ITHRCs $\mathrm{V}$ following MJ elicitation.

Factually, YUCCA can catalyze the conversion of tryptamine to $N$-hydroxytryptamine, which is acknowledged as a rate-limiting step in indole biosynthesis pathway (Suzuki et al. 2015). Apart from epigoitrin, the rest alkaloids in this work (isatin, indole-3-carboxaldehyde, tryptanthrin, indigo, and indirubin) are all indole alkaloids, which can be synthesized from the downstream metabolism of $\mathrm{N}$-hydroxytryptamine (Chen et al. 2013). Thus, the significant expression of YUCCA gene might be beneficial for the alkaloid augmentation in ITHRCs III underlying SA elicitation. Moreover, $\mathrm{CHI}$ can catalyze the stereospecific isomerization of chalcones to form (2S)-flavanones, which are critical
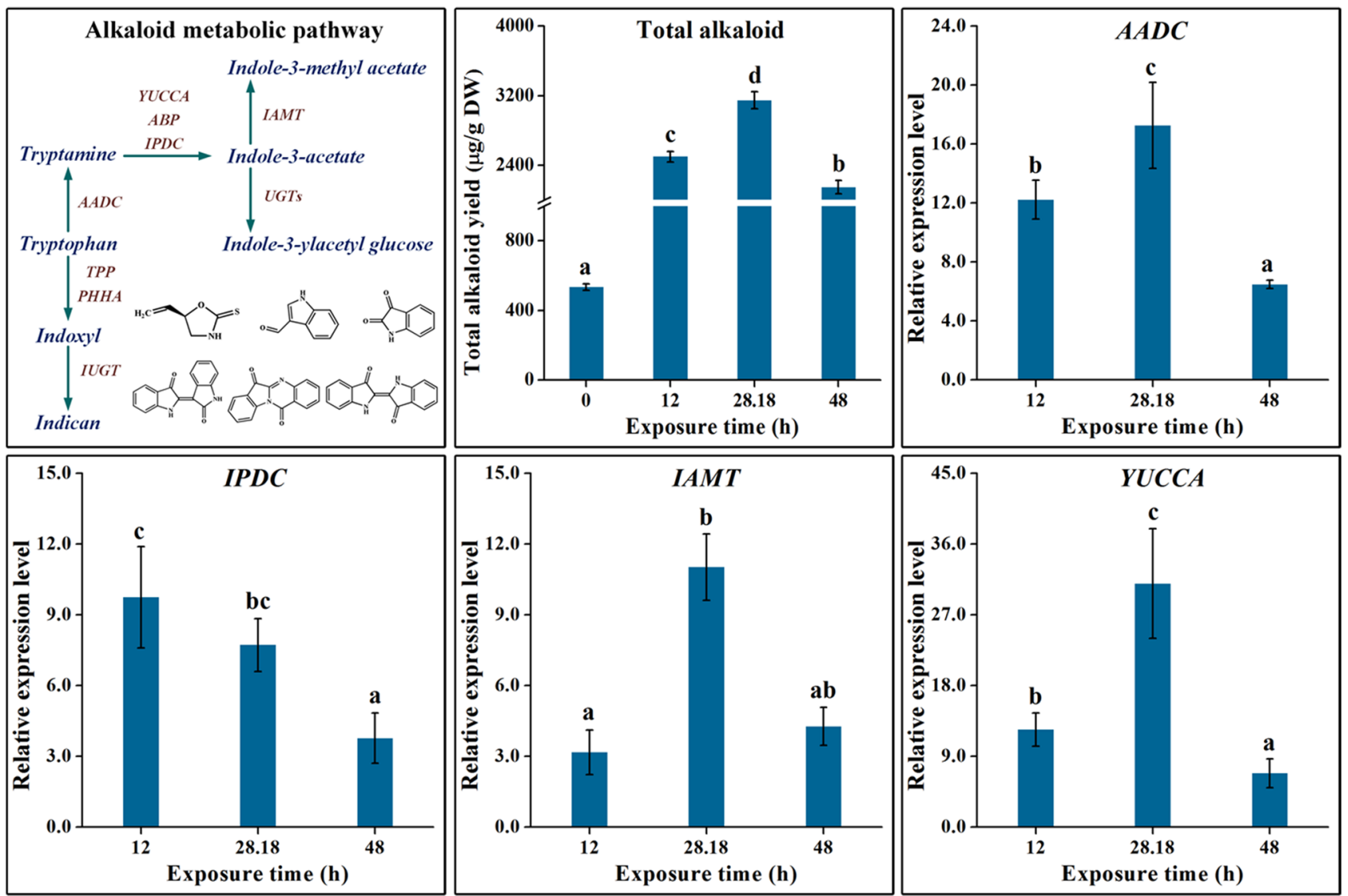

Fig. 4 Alkaloid metabolic pathway, accumulation pattern of total alkaloids, and transcriptional profiles of four alkaloid biosynthetic genes in ITHRCs III exposed to SA $(142.61 \mu \mathrm{M})$ under different elici- tation durations $(12,28.18$ and $48 \mathrm{~h})$. Mean \pm SD values not sharing the same lowercase letters are significantly different $(P<0.05)$ 


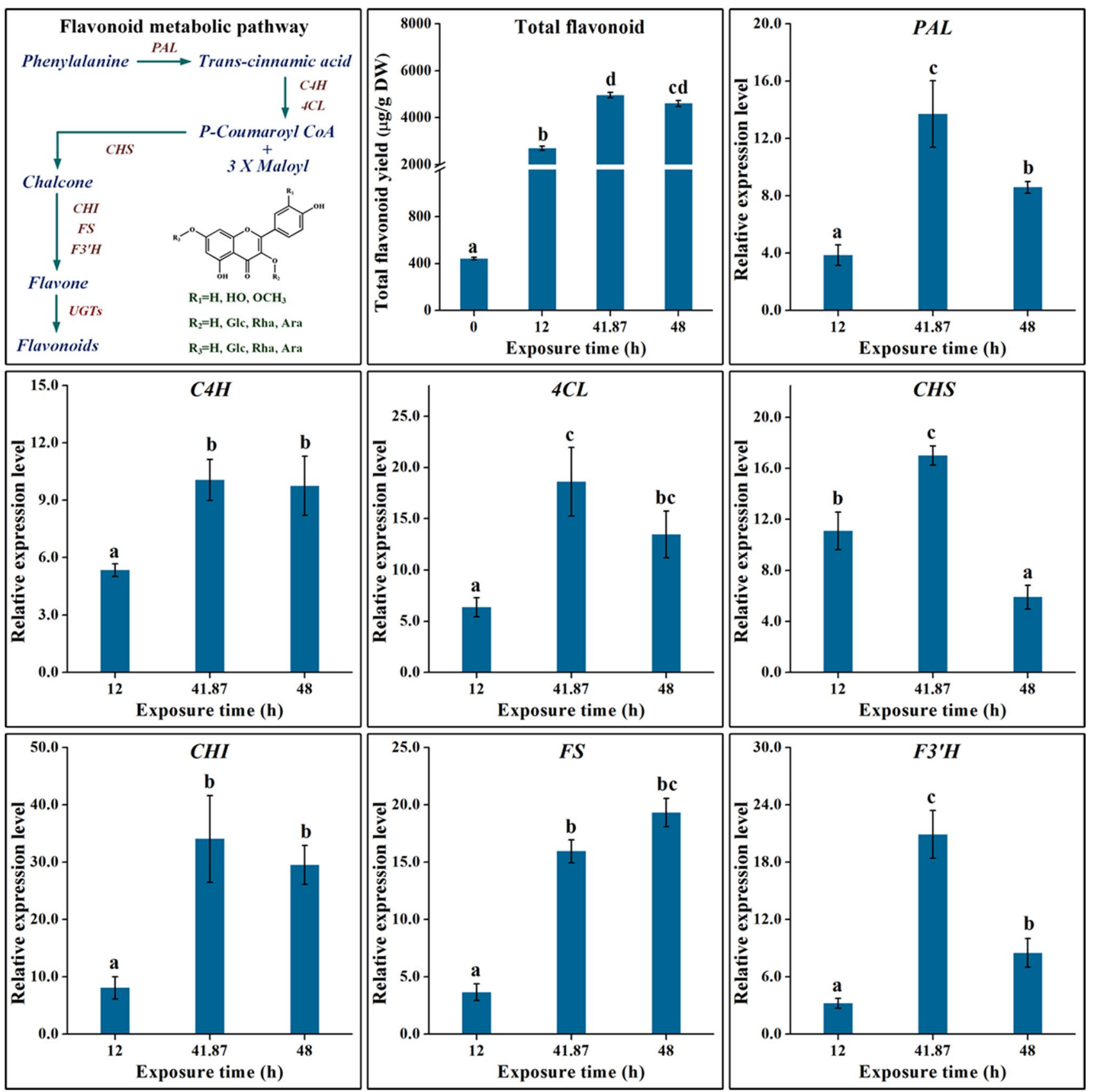

Fig. 5 Flavonoid metabolic pathway, accumulation pattern of total flavonoids, and transcriptional profiles of seven flavonoid biosynthetic genes in ITHRCs V exposed to MJ (179.54 $\mu \mathrm{M})$ under different elicitation durations (12, 41.87 and $48 \mathrm{~h})$. Mean \pm SD values not sharing the same lowercase letters are significantly different $(P<0.05)$

increase in flavonol contents. Additionally, $F 3^{\prime} H$ gene plays an essential role in the regulation of quercetin biosynthesis because its encoding enzyme can catalyze the hydroxylation of flavonoid B-ring at the 3' position (Liu et al. 2014). Accordingly, the remarkable enhancement in quercetin yield (15.97-fold increase) in ITHRCs V might be attributed to the significant induction of $F 3^{\prime} H$ transcription underlying MJ elicitation. 


\section{Conclusions}

This work demonstrated that the simple elicitation practice using exogenous SA and MJ could effectively improve the yields of pharmacologically active alkaloids and flavonoids in ITHRCs. Results showed that ITHRCs III elicited by $142.61 \mu \mathrm{M} \mathrm{SA}$ for $28.18 \mathrm{~h}$ gave a 5.89 -fold increase in alkaloid yield as compared with control, and ITHRCs $\mathrm{V}$ elicited by $179.54 \mu \mathrm{M} \mathrm{MJ}$ for $41.87 \mathrm{~h}$ caused a 11.21 fold increase in flavonoid level as against control. These findings provide useful references for the possible scaleup production of these valuable compounds by the aid of bioreactor technology in the future. Moreover, the clarification of several potential key regulatory genes (YUCCA, $C H I$ and $F 3^{\prime} H$ ) in alkaloid and flavonoid biosynthetic pathways is crucial to design metabolic engineering strategies that would enhance the biosynthesis of target phytochemicals in further studies.

Acknowledgements The authors gratefully acknowledge the financial supports by National Natural Science Foundation of China for Youths (31800492), Fundamental Research Funds for the Central Universities (2572018BU02 and 2572017DA04), Heilongjiang Province Science Foundation for Youths (QC2017012), National Key R\&D Program of China (2017YFD0600205), Scientific Research Start-up Funds for Talents Introduction of Northeast Forestry University (YQ201703), and Fundamental Research Funds for the Central Universities (2572017AA08).

Author Contributions QYG, JJ and YJF conceived and designed the experiments. QYG, JJ, and XW performed the experiments. GQY and JJ analyzed the data. GQY, XW, YPZ, and LLN contributed reagents/ materials/analysis tools. All the authors contributed to writing and editing of the manuscript.

\section{Compliance with ethical standards}

Conflict of interest The authors declare that they have no conflict of interest.

\section{References}

Ahuja I, Kissen R, Bones AM (2012) Phytoalexins in defense against pathogens. Trends Plant Sci 17:73-90

Chen J, Dong X, Li Q, Zhou X, Gao S, Chen R, Sun L, Zhang L, Chen W (2013) Biosynthesis of the active compounds of Isatis indigotica based on transcriptome sequencing and metabolites profiling. BMC Genom 14:857

Chen G, Wang S, Huang X, Hong J, Du L, Zhang L, Ye L (2015) Environmental factors affecting growth and development of Banlangen (Radix Isatidis) in China. African J Plant Sci 9:421-426

el Jaber-Vazdekis N, Barres ML, Ravelo ÁG, Zárate R (2008) Effects of elicitors on tropane alkaloids and gene expression in Atropa baetica transgenic hairy roots. J Nat Prod 71:2026-2031

Gai QY, Jiao J, Luo M, Wang W, Ma W, Zu YG, Fu YJ (2015a) Establishment of high-productive Isatis tinctoria L. hairy root cultures: a promising approach for efficient production of bioactive alkaloids. Biochem Eng J 95:37-47

Gai QY, Jiao J, Luo M, Wei ZF, Zu YG, Ma W, Fu YJ (2015b) Establishment of hairy root cultures by Agrobacterium rhizogenes mediated transformation of Isatis tinctoria L. for the efficient production of flavonoids and evaluation of antioxidant activities. PLoS ONE 10:e0119022

Giri CC, Zaheer M (2016) Chemical elicitors versus secondary metabolite production in vitro using plant cell, tissue and organ cultures: recent trends and a sky eye view appraisal. Plant Cell Tissue Organ Cult 126:1-18

Hamburger M (2002) Isatis tinctoria-From the rediscovery of an ancient medicinal plant towards a novel anti-inflammatory phytopharmaceutical. Phytochem Rev 1:333-344

Isah T, Umar S, Mujib A, Sharma MP, Rajasekharan PE, Zafar N, Frukh A (2018) Secondary metabolism of pharmaceuticals in the plant in vitro cultures: strategies, approaches, and limitations to achieving higher yield. Plant Cell Tissue Organ Cult 132:239-265

Kranner I, Minibayeva FV, Beckett RP, Seal CE (2010) What is stress? Concepts, definitions and applications in seed science. New Phytol 188:655-673

Lim W, Li J (2017) Co-expression of onion chalcone isomerase in Del/Ros1-expressing tomato enhances anthocyanin and flavonol production. Plant Cell Tissue Organ Cult 128:113-124

Lin CW, Tsai FJ, Tsai CH, Lai CC, Wan L, Ho TY, Hsieh CC, Lee Chao PD (2005) Anti-SARS coronavirus 3C-like protease effects of Isatis indigotica root and plant-derived phenolic compounds. Antiviral Res 68:36-42

Liu S, Ju J, Xia G (2014) Identification of the flavonoid 3'-hydroxylase and flavonoid 3', 5'-hydroxylase genes from Antarctic moss and their regulation during abiotic stress. Gene 543:145-152

Livak KJ, Schmittgen TD (2001) Analysis of relative gene expression data using real-time quantitative PCR and the $2^{-\Delta \Delta C T}$ method. Methods 25:402-408

Malik S, Hossein Mirjalili M, Fett-Neto AG, Mazzafera P, Bonfil M (2013) Living between two worlds: two-phase culture systems for producing plant secondary metabolites. Crit Rev Biotechnol 33:1-22

Muir SR, Collins GJ, Robinson S, Hughes S, Bovy A, Ric De Vos CH, van Tunen AJ, Verhoeyen ME (2001) Overexpression of petunia chalcone isomerase in tomato results in fruit containing increased levels of flavonols. Nat Biotechnol 19:470-474

Murthy HN, Lee EJ, Paek KY (2014) Production of secondary metabolites from cell and organ cultures: strategies and approaches for biomass improvement and metabolite accumulation. Plant Cell Tissue Organ Cult 118:1-16

Narayani M, Srivastava S (2017) Elicitation: a stimulation of stress in in vitro plant cell/tissue cultures for enhancement of secondary metabolite production. Phytochem Rev 16:1227-1252

Nguyen TKO, Jamali A, Grand E, Morreel K, Marcelo P, Gontier E, Dauwe R (2017) Phenylpropanoid profiling reveals a class of hydroxycinnamoyl glucaric acid conjugates in Isatis tinctoria leaves. Phytochemistry 144:127-140

Ramirez-Estrada K, Vidal-Limon H, Hidalgo D, Moyano E, Golenioswki M, Cusidó RM, Palazon J (2016) Elicitation, an effective strategy for the biotechnological production of bioactive highadded value compounds in plant cell factories. Molecules 21:182

Schmitz C, Fritsch L, Fischer R, Schillberg S, Rasche S (2016) Statistical experimental designs for the production of secondary metabolites in plant cell suspension cultures. Biotechnol Lett 38:2007-2014

Suzuki M, Yamazaki C, Mitsui M, Kakei Y, Mitani Y, Nakamura A, Ishii T, Soeno K, Shimada Y (2015) Transcriptional feedback regulation of YUCCA genes in response to auxin levels in Arabidopsis. Plant Cell Rep 34:1343-1352 
Tang X, Xiao Y, Lv T, Wang F, Zhu Q, Zheng T, Yang J (2014) Highthroughput sequencing and de novo assembly of the Isatis indigotica transcriptome. PLoS ONE 9:e102963

Venugopalan A, Srivastava S (2015) Enhanced camptothecin production by ethanol addition in the suspension culture of the endophyte, Fusarium solani. Bioresour Technol 188:251-257

Wang YT, Yang ZF, Zhao SS, Qin S, Guan WD, Huang QD, Zhao YS, Lin Q, Mo ZY (2011) Screening of anti-H1N1 active constituents from Radix Isatidis. J Guangzhou Univ Tradit Chin Med $4: 419-422$
Zhang HC, Liu JM, Lu HY, Gao SL (2009) Enhanced flavonoid production in hairy root cultures of Glycyrrhiza uralensis Fisch by combining the over-expression of chalcone isomerase gene with the elicitation treatment. Plant Cell Rep 28:1205-1213

Publisher's Note Springer Nature remains neutral with regard to jurisdictional claims in published maps and institutional affiliations. 